\title{
Multi-sensor Image Fusion Algorithm Based on Multiresolution Analysis
}

\author{
https://doi.org/10.3991/ijoe.v14i06.8697
}

\author{
Zhi-guo Wang $\left({ }^{\bowtie}\right)$, Wei Wang, Baolin Su \\ Sui Hua Uinversity, SuiHua, China \\ zhiguowangxt156@gmail.com
}

\begin{abstract}
To solve the fusion problem of visible and infrared images, based on image fusion algorithm such as region fusion, wavelet transform, spatial frequency, Laplasse Pyramid and principal component analysis, the quality evaluation index of image fusion was defined. Then, curve-let transform was used to replace the wavelet change to express the superiority of the curve. It integrated the intensity channel and the infrared image, and then transformed it to the original space to get the fused color image. Finally, two groups of images at different time intervals were used to carry out experiments, and the images obtained after fusion were compared with the images obtained by the first five algorithms, and the quality was evaluated. The experiment showed that the image fusion algorithm based on curve-let transform had good performance, and it can well integrate the information of visible and infrared images. It is concluded that the image fusion algorithm based on curve-let change is a feasible multisensor image fusion algorithm based on multi-resolution analysis.
\end{abstract}

Keywords—multiresolution, multisensory, image, fusion algorithm

\section{Introduction}

After years of development, the image sensor and the computing ability of computer is greatly improved. At the same time, a large number of new image sensors appear, including CCD color camera, X-ray CCD image sensor, infrared CCD image sensor, CMOS image sensor, remote sensing multispectral imager, remote sensing imaging based on SPOT, Landsat satellite imaging, millimeter wave imaging sensor, microwave imaging sensor, magnetic imaging sensor, isotope imaging sensor, X-CT imaging sensor, micro optical image sensor, acoustic imaging sensor, vehicle image sensor and process tomography sensors. Image processing has made great progress in more and more fields, and the demand for image classification and quality is increasing. However, the information obtained by a single sensor is always limited and cannot meet the requirements in all cases. Therefore, because of the shortage of single sensor imaging, multi-sensor based image fusion technology is becoming more and more widely used. For example, the spectral range accepted by the commonly used visible light images is limited to the range identified by the human eye, and the image information is far from enough. If at night or in the case of severe light, the scene 
information obtained by visible light is less. However, an infrared image can be used to image any heated objects. In the case of insufficient light, it has great superiority to the recognition of the target. Therefore, if the visible light image and the infrared image information are fused together, the information in the scene is more comprehensive.

Because there is redundancy in information between multi-sensors, it is very important to study how to remove redundant information and retain complementary information between images. The aim of this paper is to realize image fusion under curve-let transform and compare the effect of image fusion under various methods.

\section{Literature review}

In 1983, the earliest multi-resolution image fusion tools were proposed by Burt P.J and Adelson E. H, namely, Gauss Pyramid and Laplasse Pyramid. Laplasse Pyramid structure lacks directional information. According to this finding, Yuan and others as stated in [1] applied the wavelet multi resolution weight correlation image fusion method. However, the results of the contrast Pyramid and the gradient Pyramid will increase the amount of data and cause the algorithm instability when the difference of the fusion image is large. Jiang and Zhou as stated in [2] discussed the application of wavelet transform in gearbox fault diagnosis. In 90s, the proposed wavelet transform technology has good properties such as variable time frequency domain, multi-scale and directivity. It has been widely applied in the field of image fusion. Because wavelet transform has only three directions of vertical, horizontal and diagonal directions, its expression for curves is not very complete. Therefore, a series of multiresolution analysis tools such as ridgelet transform and curvelet transform have been proposed successively. Uçar and others as stated in [3] proposed a new facial expression recognition based on curvelet transform, which can effectively describe the contour of the edge curve of the image target. In 2005, Jha and Kwon as stated in [4] used Curve-let transform to fuse remote sensing image. After that, many neural network models (BP, SOFM and ARTMAP) were used for image fusion. Li and others as stated in [5] proposes a feature level fusion algorithm based on regional segmentation and neural network, which has high efficiency compared with the traditional fusion algorithm.

$\mathrm{Yu}$ and others as stated in [6] proposed a false color image fusion method based on multi-resolution color transfer in normalization. To improve the visual effect of fusion, the color image fusion method has become a hot topic of research because of the limitation of gray image. At present, scholars have put forward a number of famous color image fusion methods. The American Naval Research Lab proposed the NRL algorithm. Shi and others as stated in [7] believed that the network representation learning was suitable for tumor classification with small ultrasound image dataset. The most famous color image fusion algorithm is the MIT fusion method proposed by Sun and Jiang as stated in [8]. After that, Meng and others as stated in [9] used multisensor image fusion based on regional characteristics in mangy image fusion fields and made great achievement. In addition, Xiao and others as stated in [10] began to vigorously promote the multi-sensor image fusion algorithm. 
To sum up, the existing research in the field of image fusion algorithm is not enough. Aiming at the multi-sensor image fusion, a new image fusion algorithm based on curve-let transform is proposed to solve the fusion problem of visible and infrared images. This method makes up for the shortcomings of current research in the field of fusion and resolution analysis. Therefore, the image fusion algorithm based on curve-let change is a feasible multi-sensor image fusion algorithm based on multi-resolution analysis.

\section{Image fusion algorithm and its index}

According to the level of fusion, multi-sensor data fusion can be done at four different levels: signal level, pixel level, feature level and decision level. Signal layer fusion means that in the fusion process based on signal, the purpose is to combine the signals of different sensors and generate a new signal that is higher than the signal to noise ratio of the original signal.

Pixel layer fusion means that the fusion based on pixels is finished point by point. It produces a fusion image, and each pixel in the image is generated according to the pixel information of the multiple source images. The pixel effect promotes the superiority of the generated fusion image in the image processing (such as image segmentation).

Feature-level fusion refers to the information extraction from a variety of data sources based on the fusion need of feature. It requires extracting a significant set of features in the image, which varies according to the application domain. The commonly used parameters include pixel gray value, boundary and texture. Finally, the extracted features are fused together.

Decision layer fusion means that the information fusion in this level is high. In addition, the final fusion decision is produced by combining the results of a variety of algorithms. The input images are processed separately, and the obtained information is used for the final decision to enhance the reliability of the decision.

In multi-sensor image fusion, the most research is image fusion algorithm in pixel level. In order to fully express the information in the image and try not to lose any details of the image, multiresolution tools are used to represent the image. In the space domain, the image is decomposed by Pyramid. The original image is transformed, and the transform domain coefficients are used to represent the image. After multiresolution decomposition, the common fusion rules in the image are two fusion rules based on pixels and region based.

\subsection{Fusion algorithm based on region}

The weighted average algorithm of pixel gray value is as follows: The weight set $W_{n}(i, j)$ of the fused image $I_{n}(x, y)(n=1,2, \ldots N)$ is obtained by analysis. $N$ is the number of images to be fused, then the final fusion image $I(i, j)$ is: 


$$
\mathrm{I}(\mathrm{i}, \mathrm{j})=\sum_{n=1}^{N} W_{n}(i, j) \mathrm{I}_{\mathrm{n}}(\mathrm{i}, \mathrm{j})
$$

In the formula,

$$
\sum_{n=1}^{N} W_{n}(i, j)=1
$$

When $\mathrm{W}_{1}(\mathrm{i}, \mathrm{j})=\mathrm{W}_{2}(\mathrm{i}, \mathrm{j})=\mathrm{W}_{3}(\mathrm{i}, \mathrm{j})=\ldots=\mathrm{W}_{\mathrm{N}}(\mathrm{i}, \mathrm{j})=1 / \mathrm{N}$, the algorithm is the average algorithm of pixel gray value.

The fusion rule based on region means to select the weight coefficient of gray value of pixels by taking the characteristics of variance, gradient and energy in a pixel field as a measure. The template size of the window can be chosen by itself. Figure 1 is a schematic diagram of a fusion rule based on region. This method takes into account the correlation of pixel point domain points, and improves the fusion effect.

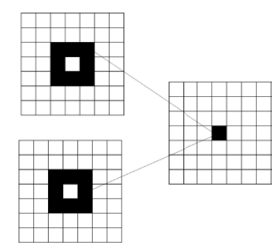

Fig. 1. Region based fusion rule diagram

For gray image fusion, first, each image $\mathrm{I}(\mathrm{x}, \mathrm{y})$ can be considered as the two part of the background image $\mathrm{I}_{\text {back }}$ and the foreground image $\mathrm{I}_{\text {fore }}$. After the fusion, the image is also processed, and then the fusion algorithm is the fusion of the foreground and background images. After the fusion, the image is also processed, and then the fusion algorithm is the foreground and background images. The background image to be fused is processed by the mean filter with the size of the window $11 \times 11$. Then, the foreground image is obtained by subtracting the background image from the original image.

\subsection{Image fusion algorithm based on spatial frequency}

Spatial frequency is a parameter that characterizes image in detail. According to this parameter, a corresponding image fusion method is proposed. It is assumed that two images to be fused are A and B. First, the images are partitioned into sub window images Block $\mathrm{A}_{\mathrm{A}}$ and $\mathrm{Block}_{\mathrm{B}}$ respectively. Then, the spatial frequency of the subimage is calculated. The spatial frequency is composed of the row frequency and the column frequency. Sub-images are convoluted with $[-1,1]$ and $[-1,1]$, respectively. Then, the sum of square after convolution is called the row frequency and the column frequency. Assuming that the fusion image is $F$, then 


$$
\operatorname{Block}_{F}(i, j)=\left\{\begin{array}{l}
\operatorname{Block}_{A}(i, j), S F_{A}>S F_{B} \\
\operatorname{Block}_{B}(i, j), S F_{B}>S F_{A}
\end{array}\right.
$$

In the formula, $\mathrm{SF}_{\mathrm{I}}$ represents the spatial frequency of the image I sub-image.

\subsection{Fusion algorithm based on image Pyramid}

The image pyramid is a multi-resolution analysis of the spatial domain structure. If we want to build the Laplacian pyramid of images, then first step is to create a Gaussian image pyramid structure. According to the bottom of the Laplasse image Pyramid, the Gauss Pyramid image of each layer can be computed and obtained by layers. In the end, the original image can be reconstructed. The image fusion method based on Laplasse Pyramid has the following steps:

Step 1: The image to be fused is decomposed into Laplasse Pyramid;

Step 2: The 1-th layer image corresponding to Laplasse Pyramid generates the fused image according to the pixel fusion rule or the region fusion rule. The new fusion image is taken as the new 1-th layer image;

Step 3: The new Laplasse image is used to restore the Pyramid layer by layer, and the final fusion image is reconstructed.

When the image Pyramid is decomposed, the amount of data often increases. Moreover, there is a correlation between the Pyramid image layers. Therefore, when the difference between the images to be fused is very large, the algorithm based on the image Pyramid will produce instability. Moreover, the decomposition of Pyramid lacks direction, and the description of the curve is neither complete nor delicate. Wavelet transform not only has the advantages of multi-resolution analysis, but also the characteristics of variable resolution in direction, space and frequency domain. Therefore, the image fusion method based on wavelet transform has received extensive attention and development.

\subsection{Image fusion algorithm based on wavelet transform}

The process of image fusion algorithm based on wavelet transform: First, the image to be fused is decomposed by wavelet transform to obtain the corresponding coefficients in the frequency domain. Then, the coefficients of the frequency domain are fused by using the corresponding fusion rules. As shown in figure 2, the fused image is obtained by inverse wavelet transform according to the coefficient of fusion.

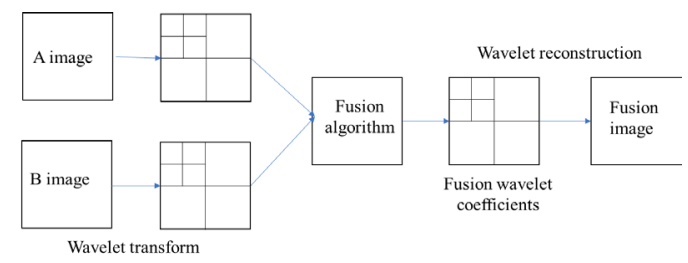

Fig. 2. Image fusion principle map based on wavelet change 
The image fusion method based on the wavelet transform has the following steps:

Step 1: A series of wavelet coefficients are obtained by the wavelet transform for the image to be fused;

Step 2: According to the image region fusion rule, the wavelet decomposition coefficient of the image in each frequency band is selected;

Step 3: The fusion image is obtained by the wavelet transform coefficient which is fused by the wavelet transform.

\subsection{Image fusion algorithm based on principal component analysis (PCA)}

As the resolution of the image sensor is getting higher and higher, the pixels of the image are getting more and more. As a result, the amount of data in the image fusion is increasing. In order to reduce the running time of the fusion algorithm, it is a feasible way to reduce the dimension of the image data. Principal component analysis (PCA) is proposed to reduce the correlation of matrix data and the dimension of data. Therefore, the image can also be regarded as a matrix composed of pixels. The fusion results can be obtained by using the PCA algorithm to extract the main component of the image.

\subsection{Image fusion index}

In order to compare the quality of all kinds of image fusion algorithms and evaluate the quality of the fused images, scholars have proposed many methods to objectively evaluate the image quality.

The image gray mean value reflects the overall brightness information of the image. If the mean is small, the overall image is very dark. If the mean value is large, the overall image is very bright. Therefore, moderate mean is the best. The standard deviation of the image gray value reflects the fluctuation of the image overall gray level near the mean value. If the standard deviation is large, the contrast of the image will be very large and the quality of the image is very good.

Entropy is the measure of the indeterminate degree of representation information in information theory. The greater the uncertainty is, the greater the entropy is, and the more information it carries. The information contained in the image before and after the image fusion will change, then the information entropy of the image can be used as an index of image quality evaluation. If the entropy of the image becomes larger after fusion, the amount of information carried by the image increases and the quality of the image is better.

The gradient characterization of the image is the change degree of image gray value, and the side reflects the details of the image. The more the detailed information is, the gradient of the image is more intense. The quality of the image is usually characterized by the average gradient of the image.

It is limited to evaluate the quality of the image fusion algorithm based on the information carried by a single image. Therefore, the information entropy of the image is extended to the combined entropy of the fusion image and the original image, and 
the joint information between the two images is represented. The greater the value of the combined entropy is, the greater the amount of information in the image is.

In the information theory, another concept is to characterize the correlation between the two variables by mutual information. The mutual information between the original image and the fused image is used as a quality evaluation index of the image. The spatial frequency represents the change of the image gradient, which is composed of the row frequency and the column frequency.

The deviation index of the image is expressed as the sum of the absolute value of the difference of the gray value of the original image and the fused image after being normalized by the gray value of the fused image. The greater the deviation index is, the worse the fusion effect is.

However, these indicators are only dependent on the fusion image to define and have some limitations. Therefore, the image quality evaluation index is obtained with the original image or the standard image as the input, and the more information is fused to a large extent. According to these indicators, the following chapters will compare the advantages and disadvantages of the fusion algorithms mentioned above and the fusion algorithm based on curve-let transform presented in this paper, and summarize the corresponding experimental results.

\section{Image fusion algorithm based on curve-let transform}

Before the image fusion is finished, some preparatory work is needed, in which many image processing techniques will be used. All of the image fusion algorithm can be divided into the following three steps: The first step is the image preprocessing. Because the fusion images are obtained from different sensors or under different conditions, there may be differences in resolution, gray range and so on. Normalization and other preliminary processing are needed. The second is the image registration. This step is an essential step in image fusion. The accuracy of registration has a direct impact on the result of the final image fusion. It is generally required to achieve one to one correspondence between the pixels of the image, and sometimes even the sub-pixel accuracy. Finally, according to the different fusion levels, the fusion image is synthesized or the feature fusion is extracted or the decision fusion is made to the results of different algorithms.

A large number of studies and scholars have shown that the wavelet transform is still defective in the representation of images. For isotropic objects, the wavelet transform can often get good results. However, the effect of anisotropic objects is not satisfactory. The most common problem is the approximation of the curvilinear contour in the image. The high frequency component of the discrete two-dimensional wavelet transform contains only three directions of vertical, horizontal and diagonal. This does not meet the requirements for the description of the curve, resulting in the loss of most of the details. Therefore, in order to solve the problem of anisotropic expression, the scholars have developed a better performance of the ridge-let transform and the curve-let transform [9]. 
The ridge-let transform is similar to the wavelet transform. A series of ridge-let functions are obtained by changing the parameters according to the function that satisfies a certain frame condition. In contrast, the ridge-let function not only has the parameters of scale and translation, but also increases the description of direction parameters adapting to the anisotropic objects. Assuming that $\varphi$ is a frame function that satisfies the condition, then it can get the ridge wave function:

$$
\varphi_{a, \theta, b}=a^{-\frac{1}{2}} \varphi\left(\frac{x \cos \theta+y \sin \theta-b}{a}\right)
$$

In the formula, a is scale parameter and $\theta$ is directional parameter. $\mathrm{B}$ is position parameter. For any square integrable function $\mathrm{f}$, the corresponding ridge-let coefficients are as follows:

$$
R_{f}(a, \theta, b)=\left\langle f, \varphi_{a, \theta, b}\right\rangle=\int f(x) \overline{\varphi_{a, \theta, b}} d x
$$

In the formula, $\overline{\varphi_{a, \theta, b}}$ represents the complex conjugation of $\varphi_{a, \theta, b}$. The available coefficients of $\mathrm{f}$ are completely reconstructed.

$$
f=\int_{0}^{2 \pi} \int_{-\infty}^{\infty} \int_{0}^{\infty} R_{f}(a, \theta, b) \varphi_{a, \theta, b}(x) \frac{d a}{a^{3}} d b \frac{d \theta}{4 \pi}
$$

The ridge-let transform often uses the discrete form, so three parameters are discretized. The formula (7) is obtained according to the definition of the $\mathrm{L}^{2}$ framework:

$$
\forall f \in L^{2}, \exists A, B>0
$$

Then, the formula (8) is obtained:

$$
A\|f\|_{L^{2}}^{2} \leq \sum_{j .1 . k}\left|<f, \varphi_{j .1 . k}>\right|^{2} \leq B\|f\|_{L^{2}}^{2}
$$

Then, $\mathrm{f}$ is always reconstructed by the coefficient set of ridge-let transform $\left\{<f, \varphi_{j .1 . k}>\right\}_{j, k, l \in Z}$.

Relative to the superiority of wavelet, the ridges are directional. The direction parameter $\theta$ is introduced outside the scale and position parameters, and its discretization is as follows:

$$
\theta_{j, 1}=2 \pi \theta_{0} 12^{-j}
$$

The discretization method decomposes the two-dimensional plane of the frequency into several rings. Within the $2^{\mathrm{j}} \leq \mathrm{a} \leq 2^{\mathrm{j}+1}$, it was also divided into $2^{\mathrm{j}}$ sub-regions. The 
discrete ridge-let transform can be realized by Radon transform, and the Radon transformation in the two-dimensional space is expressed as:

$$
R f(\theta, t)=\iint f\left(x_{1}, x_{2}\right) \delta\left(x_{1} \cos \theta+x_{2} \sin \theta-t\right) d x_{1} d x_{2}
$$

In the formula, $\delta$ represents the Dirac function. Then, the ridge-let transform can be performed by two steps of Radon transform and wavelet transform:

$$
\left\langle f, \varphi_{j, k, 1}\right\rangle=\left\langle\operatorname{Rf}(\theta j, 1, \bullet), \varphi_{j, k}\right\rangle
$$

In the formula, $\varphi_{j, k}$ represents the wavelet function. The process of ridge-let transform is shown in figure 3.

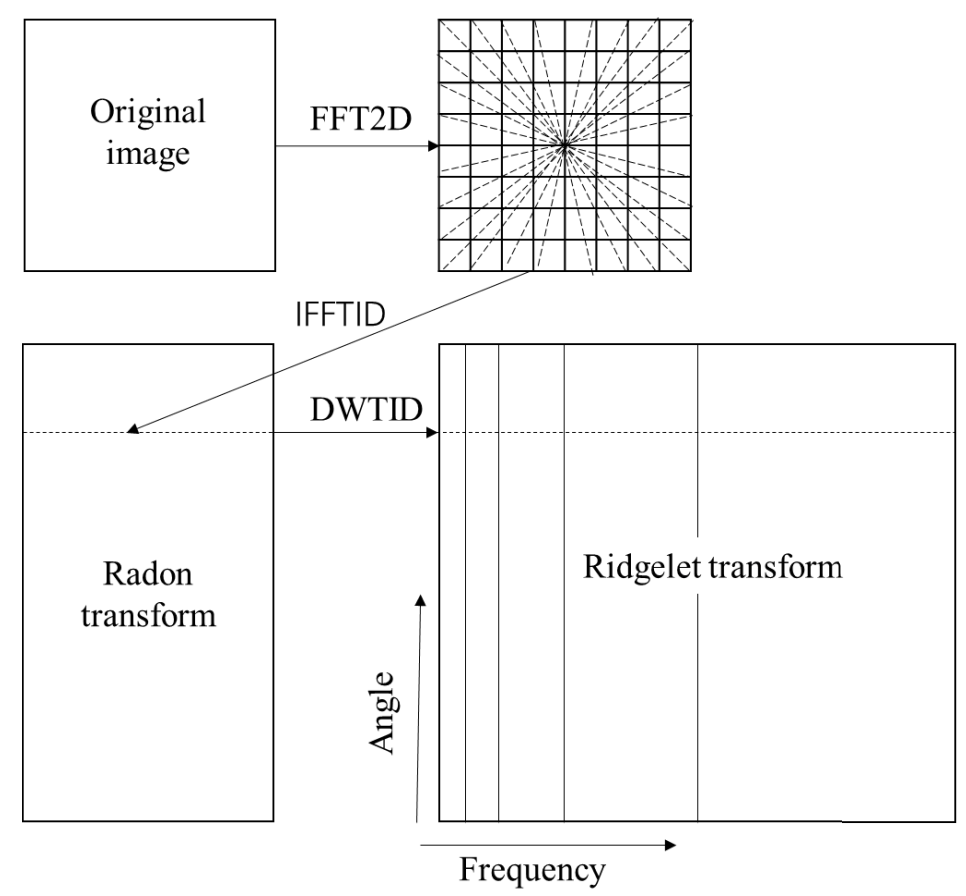

Fig. 3. Ridge-let transform flowchart

The curve-let transform is essentially a local ridge wave transformation under multiscale. The local ridge-let transform is a single scale, which divides the image into many sub blocks. Therefore, each block can be approximated by a straight line, and then the ridge-let transform is used for the straight line segments. The curvelet transform first achieves the multi-scale sub-band segmentation by using wavelet transform, and then the ridge-let transform is carried out on the appropriate scales for each subband. The main steps of the curve-let transform for images are shown in figure 4. 


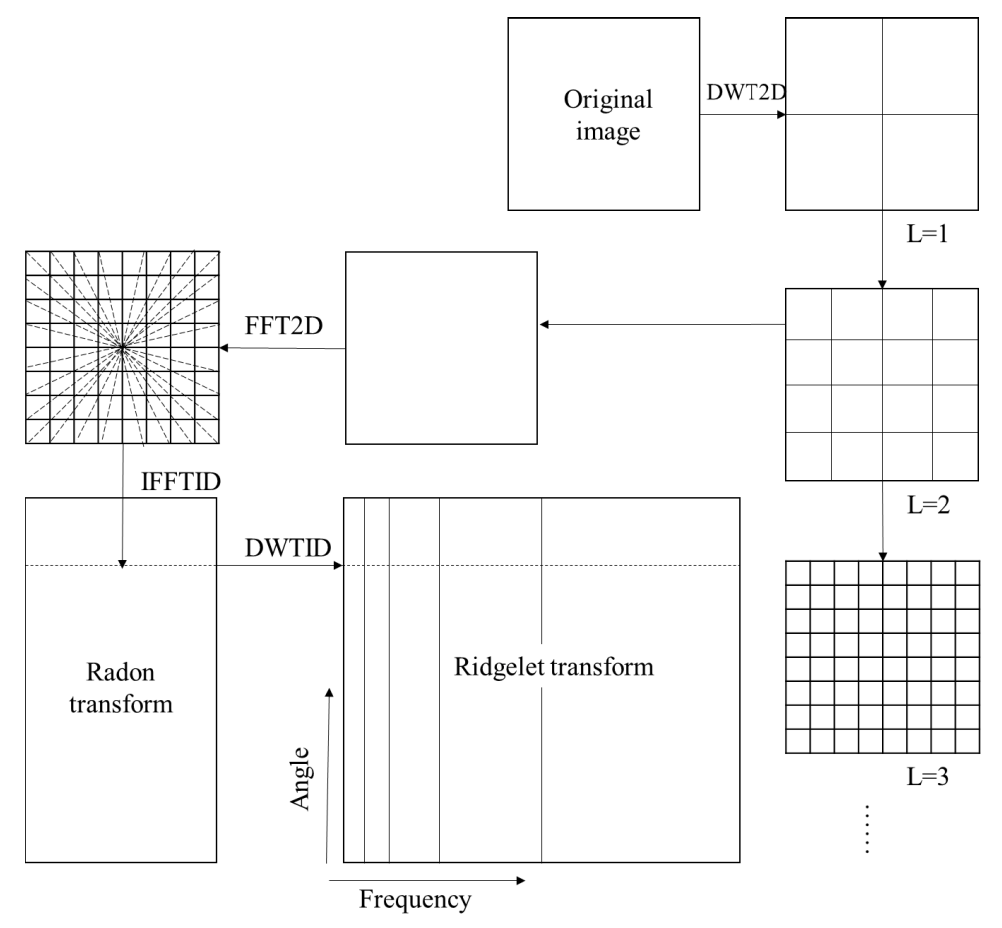

Fig. 4. Curve transform flowchart

Assuming that I is an image with the size of $\mathrm{N} \times \mathrm{N}$. Then, after the wavelet transform is used for J-th layer, the approximation of the image can be obtained:

$$
I=C_{J}+\sum_{J=1}^{J} D_{J}
$$

In the formula, $\mathrm{C}_{\mathrm{J}}$ is the approximate component when the scale is $\mathrm{J}$, and DJ is the detail component of each scale. On the basis of the above hypothesis, the discrete curve-let transform can be realized.

The image fusion based on curve-let transform is similar with the wavelet transform. In contrast, the curve-let transform is more directional than the wavelet transform, and can better retain the details of the image. Therefore, its performance is also more superior [10]. First, the image to be fused is transformed by curve-let. Then, the obtained curve-let coefficients are fused in accordance with certain rules. Finally, the fused image is reconstructed based on the fusion curve-let coefficient. The purpose of this paper is to solve the fusion between visible and infrared images. The infrared image is generated in the form of gray-scale. It converts visible light to HSV color space and fuses brightness channel and infrared image. 


\section{Experimental results of image fusion}

This paper deals with the fusion of visible light color and infrared images. However, infrared images are usually kept in gray-scale. There are two different ways of thinking. The first is to convert the visible light image into the gray image directly, and then fuse with the infrared image. The second is to transform the visible light image into another color space, and extract the single channel representing the intensity and fuse with the infrared image. Finally, the color images of the RGB space are rebuilt. Considering that the first method will lose most of the hue information, the fusion effect is not very ideal. In this paper, the second idea is applied to transform the image in RGB space to the HSV space fusion that accords with the human eye observation system.

HSV color space is based on the principle of human visual imaging system, which describes color with hue, color saturation and value. It is closer to the human visual characteristics than the RGB color space. Furthermore, the separation of tone and light intensity is beneficial to the realization of many image algorithms, which greatly simplifies the workload of image processing [11].

HSV color space and RGB color space can be converted to each other through the formula (13):

$$
F=\frac{2 R-G-B}{G-B}
$$

In order to show the reliability of the algorithm, the images taken from two groups of different periods are fused. One group is a visible light image and an infrared image obtained during the day, and the other is obtained in the evening. The visible and infrared images of the experiment are images that have been accurately matched in the same scene. In the daytime, the image fusion experiment of curve-let transform shows that the original hills, poles and left buildings in the visible images can be displayed in new color images after fusion. Moreover, this information is provided in the infrared image of the same time period. Table 1 shows the comparison of the other five fusion methods and the curve-let transform fusion.

Table 1. Contrast of image quality index in daytime

\begin{tabular}{|c|c|c|c|c|c|}
\hline Method & Mean value & Entropy & Cross entropy & $\begin{array}{c}\text { Peak signal to } \\
\text { noise ratio }\end{array}$ & Joint entropy \\
\hline CEMIF & 230.2844 & 3.2539 & 0.78725 & 30.9666 & 4.7556 \\
\hline SF & 233.5724 & 3.217 & 0.70361 & 32.1768 & 4.0933 \\
\hline LP & 235.9449 & 3.0358 & 0.75988 & 31.4608 & 4.3568 \\
\hline Wavelet & 244.098 & 2.8498 & 0.7851 & 35.4226 & 4.2304 \\
\hline PCA & 234.9727 & 3.3395 & 0.92936 & 31.1111 & 4.5594 \\
\hline Curve-let & 240.4305 & 4.5336 & 0.75112 & 32.2806 & 5.7984 \\
\hline
\end{tabular}


As shown in table 1, the entropy and joint entropy of the fused image based on curve-let transform are better than the other five fusion algorithms. The mean of the image is second only to the result of wavelet transform. However, the cross entropy is only a bit worse than the algorithm based on spatial frequency, and is located in the second of the six fusion algorithms. The peak signal to noise ratio of the image is also better. In a comprehensive view, the fusion algorithm based on curve-let transform in HSV color space is superior to the other five fusion algorithms.

Based on the fusion results of a group of images at nighttime, it shows that the four wires that were not shown in the optical image could be displayed after fusion. At the same time, the hills that have been blurred in the original visible image are clearly displayed after the fusion. Table 2 shows the comparison of the other five fusion methods and the curve-let transform fusion.

Table 2. Contrast of image quality index in the evening

\begin{tabular}{|c|c|c|c|c|c|}
\hline Method & Mean value & Entropy & Cross entropy & $\begin{array}{c}\text { Peak signal to } \\
\text { noise ratio }\end{array}$ & Joint entropy \\
\hline CEMIF & 201.2788 & 6.1819 & 1.5246 & 30.3083 & 11.7579 \\
\hline SF & 168.7545 & 6.7102 & 1.3152 & 35.96 & 8.3706 \\
\hline LP & 184.0328 & 6.4723 & 1.0027 & 30.7131 & 11.2195 \\
\hline Wavelet & 214.8105 & 6.2097 & 1.1684 & 35.3128 & 11.5957 \\
\hline PCA & 193.0061 & 6.2313 & 1.4186 & 30.3502 & 10.6861 \\
\hline Curve-let & 206.3996 & 6.952 & 1.0696 & 31.0653 & 11.895 \\
\hline
\end{tabular}

As shown in table 2, the two indexes of image entropy and joint entropy obtained by curve-let transform fusion are preceded by other fusion algorithms, and all the other indicators have a good performance. Based on the fusion experiment of two sets of images in two periods, and the comparison of performance indicators, based on curve-let transform image fusion of HSV color space, the information of visible and infrared images is well integrated. However, the gray level of the infrared image is low gray as a whole. Therefore, the overall brightness of the fused visible light image has decreased. Therefore, the fusion of gray drawing and image can be considered by the infrared image.

\section{Conclusions}

Based on image fusion algorithm, the quality evaluation index of image fusion was defined. Then, curve-let transform was used to replace the wavelet change to express the superiority of the curve. The experiment showed that the image fusion algorithm based on curve-let transform had good performance. According to the analysis result, the following views can be achieved:

First, the fusion problem of visible and infrared images is to be solved in this paper. Five kinds of image fusion algorithms based on wavelet transform, spatial frequency, Laplasse Pyramid, principal component analysis and foreground background are introduced. 
Then, considering the limitations of the wavelet transform and the characteristics of the infrared image, the fusion problem of the RGB space visible light image is converted to the HSV color space. The superiority of curve-let transform to the curve expression is proposed to replace the wavelet transform. The intensity channel and the infrared image are fused and then converted to the RGB space to obtain the fused color images.

Finally, the images of two groups at different periods are used to carry out the experiment, and the quality evaluation for the images obtained after the fusion is carried out. Comparing the experimental images obtained by the six fusion algorithms, it is found that the curve-let transform fusion algorithm based on the HSV space is superior in performance. Therefore, it is a feasible image fusion algorithm to integrate the information of visible and infrared images.

\section{$7 \quad$ References}

[1] Yuan, X., Li, C., \& Sheng, H. Wavelet multi resolution weight correlation image fusion method. Journal of Beijing University of Aeronautics \& Astronautics, 2013, vol. 39(6), pp. 847-852.

[2] Jiang, C., \& Zhou, X. Application of laser self-mixing interference technology and wavelet transform in gearbox fault diagnosis. Optical Technique, 2017, vol. 43(1), pp. 83-86.

[3] Uçar, A., Demir, Y., \& Güzeliş, C. A new facial expression recognition based on curvelet transform and online sequential extreme learning machine initialized with spherical clustering. Neural Computing \& Applications, 2016, vol. 27(1), pp. 131-142. https://doi.org/10.1007/s00521-014-1569-1

[4] Jha, D., \& Kwon, G. R. Alzheimer disease detection in mri using curvelet transform with k-nn. Journal of Korean Institute of Information Technology, 2016, vol. 14(8), pp. 121. https://doi.org/10.14801/jkiit.2016.14.8.121

[5] Li, X., Liu, M. M., Xu, Y. F., \& Luo, C. A recognition algorithm of generic objects based on feature-level fusion of $2 \mathrm{~d}$ and $3 \mathrm{~d}$ sift descriptors. Chinese Journal of Electronics, 2015, vol. 43(11), pp. 2277-2283.

[6] Yu, X., Ren, J., Chen, Q., \& Sui, X. A false color image fusion method based on multiresolution color transfer in normalization yc b c r, space. Optik - International Journal for Light and Electron Optics, 2014, vol. 125(20), pp. 6010-6016.

[7] Shi, J., Zhou, S., Liu, X., Zhang, Q., Lu, M., \& Wang, T. Stacked deep polynomial network based representation learning for tumor classification with small ultrasound image dataset. Neurocomputing, 2016, vol. 194(c), pp. 87-94. https://doi.org/10.1016/ j.neucom.2016.01.074

[8] Sun, Y., \& Jiang, L. Color multi-focus image fusion algorithm based on fuzzy theory and dual-tree complex wavelet transform. Journal of Algorithms \& Computational Technology, 2017, vol. 11(2), pp. 174830181668968. https://doi.org/10.1177/1748301816689686

[9] Meng, F., Shi, R., Shan, D., Song, Y., He, W., \& Cai, W. Multi-sensor image fusion based on regional characteristics. International Journal of Distributed Sensor Networks, 2017, vol. 13(11), pp. 155014771774110. https://doi.org/10.1177/15501477177411105

[10] Xiaobing, Z., Wei, Z., \& Mengfei, S. Oil exploration oriented multi-sensor image fusion algorithm. Open Physics, 2017, vol. 15(1), pp. 188-196. https://doi.org/10.1515/phys2017-0020 
Paper-Multi-sensor Image Fusion Algorithm Based on Multiresolution Analysis

\section{Authors}

Zhi-guo Wang, Wei Wang, and Baolin Su are with Sui Hua Uinversity, SuiHua 152061, China.

Article submitted 23 January 2018. Resubmitted 09 March 2018. Final acceptance 18 March 2018. Final version published as submitted by the authors. 